\title{
The next step for Artificial Intelligence in a globally connected economy
}

\author{
Dumitru Alexandru Bodislav ${ }^{1, *}$, Florina Bran ${ }^{1}$, Carol Cristina Gombos ${ }^{2}$, and Amza Mair ${ }^{3}$ \\ ${ }^{1}$ Bucharest University of Economic Studies, Romania \\ ${ }^{1} \mathrm{PhD}$ Student, Bucharest University of Economic Studies, Romania \\ ${ }^{3} \mathrm{PhD}$ Student, Valahia University of Targoviste, Romania
}

\begin{abstract}
Research background: This research paper represents an overview of what artificial intelligence is, what are its roots, and what is the next big thing regarding the domain. In this paper we try to highlight how the domain is growing and what is the difference between the ideology, the business factor and the human factor. We try to create a big picture on the entire phenomenon by creating a parallel between machine learning, artificial intelligence and the influence of technological breakthrough from a hardware perspective.

Purpose of the article: The paper is built as a tool in understanding technology, globalization and the pathway to success and scientific glory for what can be seen as the industry of artificial intelligence. The tools presented in the research have the purpose to create an easier path to how we can develop this domain by accelerating theoretical processing and business analytics that come together to form the next level of machine learning/artificial intelligence; research and development, everything being filtered from an economic point of view.

Methods: The used research method is based on fundamental analysis of the artificial intelligence domain and its purpose in the complexity of globalization and economic development.
\end{abstract}

Findings \& Value added: The paper tries to offer a tool for building a better understanding of the next decade in the domain of artificial intelligence.

Keywords: artificial intelligence; economic development; technology

JEL Classification: $011 ; 032$

\footnotetext{
* Corresponding author: alex.bodislav@ase.ro
} 


\section{Introduction}

We aim to obtain an intimate view on human behaviour and what it means to live a feeling, or rather to develop a feeling, from both a public and private standpoint (Androniceanu and Burlacu, 2017). Corporations use a variety of analysis techniques, such as crowdsourcing, genetic algorithms, sentiment analysis, and neural networks, to study the data that drives an individual's evolution and can be used to develop new products or processes, as well as macroeconomically outlining new work policies (Balu et al, 2021). Data was gathered from a variety of sources (Ionita and Burlacu 2007). Government databases, online commerce databases, online profiles (Facebook, Weebo, etc.) and char sensors used in cell phones and wristbands are all examples.

Data mining is a new cybernetic idea, although data analysis has existed from the earliest statistical records (meteorological data), reflecting an early version of Big Data (Burlacu and Jiroveanu, 2011), and the depiction of increasingly bigger data series merely indicates its qualitative frequency (Pricop et al., 2016). Data streams, also known as data rivers, are large amounts of data that are constantly flowing and are effectively saved and rearranged to give real-time ideal solutions throughout a product's or company's life cycle (Ioniţă and Burlacu, 2007).

\section{Methodology - Big Data and Machine Learning}

Since the Internet's inception, the process of transitioning from a text-based communications economy to a much more complicated data stream based on picture, video material, and metadata linked to location, date, and mood has grown into a considerably more complex data stream (Orzan et al., 2020). The growth of WIFI and 5G enabled a new generation of the unlocated social, and human-machine internationalization became a bidirectional hybrid telemetry, resulting in the connection between computerized health networks that allow medical data synergy (radiographs, CT scans and MRI).

Because enormous amounts of data are saved file by file to meet a continuous and realtime analysis in the goal to give proactive answers, data storage is a challenge in reverse data mining. Virtual onsite shopping solutions will be deployed in Romania based on the user's mood and position within a mall (Michael and Clarke, 2013).

Big Data may also be used to reveal in real time hidden subconscious behavioural tendencies and even predict intentions (from the clear example of the anticipation of epidemics by Google Analytics to the anticipation of a crime in the not too distant future Minority Report, 2002). Simultaneously, behavioural mapping aids in the connection of the individual with his aspirations in advance, as well as a social standing that is appropriate for his requirements. The information obtained and rationally aggregated assists government bodies and companies in making clear judgments about safeguarding and preserving a people's integrity while guaranteeing the functional support of individual requirements.

Patient data aids the medical system in finding speedier treatments for illnesses like cancer and preventing the emergence/progression of others (Human Genome Project - 2003, Human Brain Initiative - 2013, Strickland, 2013). Work is underway to create a computer that follows the logic of the human brain and simulates 100 billion neurons (the number of neurons in an ordinary standard brain), with the goal of finding answers to Parkinson's and Alzheimer's disease.

Although Big Data delivers information on a variety of topics, it also has evolution and storage issues, with the cost and security of this data being the primary concerns. 


\section{Results}

\subsection{Step by Step Corporate Positioning}

The successful listing of the GoPro firm demonstrates not only the close access to video footage and its usage as a component of Big Data, but also the occupation of the level of information storage (high volume storage), demonstrating the focus on objective video content (Santos and Eisenhardt, 2005). Surveillance cameras in buildings, public spaces, and police cars represented the first wave of objective video data, with the second wave being the content created by anyone who attaches a camera to a car, bicycle, motorcycle, helmet, or other object, and thus the new content created can be used as Data in the information process, with its aggregation at the macro level creating Big Data, which is subject to the issuing of licenses.

Another issue is the moral and ethical use of Big Data, because while some companies use data to learn (through machine learning and Artificial Intelligence) how to increase productivity and rebuild work processes, they can be struck individually by the fact that it is measured quantitatively against his co-workers in the same office or against colleagues in the opposite hemisphere of the globe (Rochet and Tirole, 2003). The Internet of Things, along with the synergy between surveillance technologies as an asset or as an entity, might lead to a situation where the individual becomes a continuous video stream (Google Glasses, GoPro Hero 9, etc.) and thus Big Data content grows rapidly. The issue of privacy protection complements the situation's morals and ethics by the fact that every online and physical user generates a fingerprint accidentally, and by aggregating all means of introduction forms a digital DNA that cannot be observed otherwise (McIntyre, 2011). This evolutionary behavioural map can be built through online posts, discrepant use of punctuation depending on the condition, clothes worn depending on the event, and how to spend your free time, and it can be stored in the background latently, passively, and with an interruption of the data feed according to the graph, which could lead to an automatic alert of the authorities to be able to intervene in case of need. Meso-familial data shows evolutionary and predictive trends on people, such as the usage of internet data volume and power use.

Even if individuals alter their online profiles to protect their identities and "skip" certain validations, advanced marketing that segments the market and offers products related to the needs can insist on creating a super-supervisory company with the help of Big Data; this society can evolve towards stopping any type of crime while also evolving towards a high level of surveillance. The good aspect is that the advancement of Big Data analytics will lead to the creation of ultra-screening outcomes. early ones, with these being genetically prevented from evolving (Alzheimer's illness is a good example).

Some classical economists and physical business theorists believe that data in the form of large volumes (Big Data) is a temporary issue in the global economic circuit, but these large volumes of data have already become fundamental technological changes that have emerged and stabilized as part of the decision-making circuit, not just inside top firms like Google, Facebook, Amazon, or Microsoft (Michael and Miller, 2013), but also among those who promote museums (Smithsonian, Guggenheim, etc.) or states through hybridizing David Ricardo's notion of comparative advantage (Teece, 2012, Michael and Miller, 2013). (Singapore, Chile, etc.).

Big Data as a support for specific choices is not a new idea, but it is not a component of the IT industry under the umbrella of business analytics, which has been reinforced and accelerated by the usage of Artificial Intelligence. The difference between these concepts is that today's low cost of collecting, storing, and analysing data allows the demographic component (location, sex, age, social status, etc.) to be integrated into the decision-making process, as well as the geographical and evolutionary structuring of owned product stocks 
(Xiaohui et al., 2020). This circuit is valid for medium and large retailers, for example: the implementation of CoraDrive by the retailer Cora was done following the construction of a Business Intelligence solution that was dynamized by using Big Data.

\subsection{Business Analytics and the Push Forward on Technological Globalization}

The conventional idea of business analytics is built on three pillars: descriptive, predictive, and prescriptive. Descriptive means explaining a phenomena and presenting data in a static style (Rochet and Tirole, 2006). Predictive analytics uses historical data to create a forecast for the future and is found in all businesses, regardless of their novelty or size, prescriptive analysis is extremely important in the decision-making circuit, with the role of optimizing and creating recommendations, and the key $\operatorname{cog}$ in the decision-making circuit is predictive analytics.

However, in this study, we look at the decision automation component, which puts pressure on the descriptive and predictive components when a human evaluator is present. When the prescriptive component is related to the idea of decision automation, particularly if a decision model has been built a priori.

The use of data can be traced back to ancient times in both historical and statistical terms, with China, the Roman Empire, and Ancient Greece promoting the feasibility of using data in conjunction with macroeconomic policies and a general desire to improve welfare within the empire or state in question. To further appreciate the decisional novelty, consider the situation of a publisher who needs to decide on the distribution of a new novel; if he utilized traditional business analytics, the result would be an assessment of the sales trend for that category and calendar time maximum point in book sales) and thus, with the help of a statistical analysis based on a series of time, would achieve the necessary copies to order from that volume, completing the components of descriptive and predictive analysis, and the addition of prescriptive analysis would result in an evaluation of the future income and profit resulting from the newly released novel, however, the inclusion of Big Data examined using Business Intelligence filters leads in other components studied in connection to the demand for that innovation being added to the statistical analysis of sales. The future is not as secure as statistical analysis suggests; if it were, we would only have to deal with simple systems like a person falling from the 10th floor of a building; knowing that gravity is the only agent, we can calculate almost exactly where that person is after 2 seconds (even if there are deviations such as wind direction and speed or the weight of the person), However, when dealing with complicated systems that aren't well-defined and don't have all of the forces available to quantify, we have to rely on sophisticated econometric evaluations of large errors, or even self-correlation.

The analysis of demand for a good, the evolution of a possible epidemic, or the simple behaviour of Antarctic glaciers is evolutionary complexity between present and future filtered by unknown elements a priori, so it can be argued that the future is a function of the present, such as if you are on the subway and someone sneezes at you, your chances of catching a cold are extremely high, an example of this is if you are on the subway and someone sneezes at you and Big Data is now self-validating as a practicality and importance by using volume, diversity, veracity, and speed as characterisation aspects of the collected data (Gawer and Cusumano, 2002, 2008).

The characteristics of Big Data (speed, variety, volume, and veracity) are the most important factors in defining the components that give life to the adaptive system provided by Big Data (Jacobides, 2008). We must deal with the following defining aspects inside the three components:

1. Volume - data size (GB, TB, $\mathrm{PB}$ ), total data recording, data flow trading, and sorting in files and work tables are all examples of volume. 
2. Speed - workload, creation time, global construction time, and produced and utilized virtual data flows;

3. Data that is organized, unstructured, or semi-structured.

\section{Discussion and Conclusion}

Currently, descriptive, predictive, and prescriptive analysis are required to extract the essence of Big Data. Classic tools, on the other hand, are no longer relevant when the volume of data is too large; from personal experience, querying a sheet in Excel (Microsoft Office) with over 65,000 information lines and 10 columns of information about each line results in the file frequently failing to open, even with the processing power of the hardware component.

Hadoop, a scalable platform that can dynamically filter large volumes of data (macro Big Data) while also providing the necessary filtering for real-time dynamization of this data, whose flow and data tree logical dependencies to be filtered by software solutions such as MapReduce, a solution that also reduces repeatability, is a working solution that solves the problem of data variety (Russom, 2011).

Hospitals (patient records are electronically recorded, and background analysis can reveal other health hazards or possible future complications), retailers (Walmart uses targeted marketing by mining consumers, and Carrefour uses the physical behaviour of consumers in space), and tax services are all using Big Data as a decision-maker (the US Treasury uses fraud identification algorithms). All of the algorithms described are part of the "machine learning" component, which emphasizes learning from past mistakes and avoiding them in the future.

The value of integration into an ecosystem is given by IT solutions that can create the basic link between big data and shaping a flow from part to whole between decision and potential growth, with the best solutions being offered by Cloudera, EMC, IBM, Impetus Technologies, Oracle, Kognitio, Actian, SAND Technology, SAP, SAS, Tableau Software, Teradata, and others.

The fundamental features of Big Data's utilization in industries, as well as an examination of the theory-practice link via the application of Big Data Analytics principles at the microeconomic and macroeconomic levels (Russom, 2011).

Macroeconomic limitations that diminish sawtooth developments between Aggregate Demand and Supply are feasible, as can overdevelopment of production facilities, therefore optimizing technical production yields must meet the requirements of at least medium-term predicted markets. Reflections and next steps Big Data and Business Intelligence are intangible components of the future that have connected mankind from the industrial to the information eras (Bodislav, 2015). To understand the phenomenon's value, we must first identify the problems that may arise from the specific extraction of this data from the public sector (even without quantifying some people involved in the process as data carriers and creators - the population of a country or economic union) and offering it as a work unit, because these technologies are still in their infancy and may have the potential to provide us with ways to improve our standard of living through connectivity (efficient medical system, shaped labor market education, and national values that provide and maintain that nation's comparative advantage, proactively enhanced national security, respect for privacy, and strengthening the respect and applicability of the law, and merging a person's virtual and physical existence), growing the economy through decision-making independence, and providing pride to people of a country to be a part of that country. 


\section{References}

1. Androniceanu, A., \& Burlacu, S. (2017). Intelligent system for assessment and grading based on docimologic tests. eLearning \& Software for Education, 2.

2. Balu, F. O., Radulescu, C. V., Bodislav, D. A., Gole, I., Buzoianu, O. C. A., Burlacu, S., \& Balu, P. E. (2021). Cost modeling and computation in the healthcare industry. Case study on a Swiss medical care organization. Economic Computation \& Economic Cybernetics Studies \& Research, 55(1), 73-88.

3. Bodislav, D. A. (2015). The Synergy between Big Data - Business Intelligence. The Solution for Creating Relevant Indicators for an Emerging Economy, ASE Publishing.

4. Bodislav, D. A. (2016). Business intelligence for decision making in economics, chapter in Dunis, C., Middleton, P., Karathanasopolous, A., Theofilatos, K. \& Bodislav, D. A. Artificial Intelligence in Financial Markets. New Developments in Quantitative Trading and Investment, Palgrave Macmillan.

5. Burlacu, S., \& Jiroveanu, D. C. (2011). The develop of software solution for supply chain management. Review of International Comparative Management, 12(Special number 1), 140-145.

6. Gawer, A., \& Cusumano, M. A. (2002). Platform Leadership: How Intel, Microsoft and Cisco Drive Industry Innovation, Harvard Business Review Press.

7. Gawer, A., \& Cusumano, M. A. (2008). How companies become platform leaders. MIT Sloan Management Review, 49, 28-35.

8. Ioniţă, F., \& Burlacu, S. (2007). Macroeconomic policy in the newest e.u. member states under the impact of globalisation. Chances and Threats of the European Union for Romania, 2nd International Conference Globalization and Higher Education in Economics and Business Administration. Alexandru Ioan Cuza University of Iaşi, Faculty of Economics and Business Administration, Iaşi.

9. Jacobides, M. G. (2008). How capabilities differences, transaction costs, and learning curves interact to shape vertical scope. Organizational Science, 19, 306-326.

10. McIntyre, D. P. (2011). In a network industry, does product quality matter?, Journal of Product Innovation Management, 28, 99-108.

11. Michael, K., \& Clarke, R. (2013). Location and tracking of mobile devices: Uberveillance stalks the streets. Computer Law \& Security Review, 29, 216-228.

12. Michael, K., \& Miller, K. (2013). Big data: New opportunities and new challenges. IEEE Computer Society, 6, 22-24.

13. Orzan, M. C., Burlacu, S., Florescu, M. S., Orzan, O. A., \& Macovei, O. I. (2020). The effects of online marketing on financial performance in the textile industry. Industria Textila, 71(3), 288-293.

14. Pricop, L. C., Burlacu, S., \& Demeter, M. L. (2016). Managing violence in health sector through bi solutions. Proceedings of the International Management Conference, 10(1), 512-519.

15. Rochet, J. C., \& Tirole, J. (2003). Platform competition in two-sided markets. Journal of the European Economic Association, 1, 990-1029.

16. Rochet, J. C., \& Tirole, J. (2006). Two-sided markets: A progress report. The RAND Journal of Economics, 37, 645-667.

17. Russom, P. (2011). Big data analytics. The Data Warehousing Institute Best Practices Report, 4th Quarter. 
18. Santos, F. M., \& Eisenhardt, K. M. (2005). Organisational boundries and theories of organization. Organization Science, 16, 491-508.

19. Strickland, E. (2013). The Gene Machine and Me, IEEE Spectrum, 3, 26-32.

20. Teece, D. J. (2012). Dynamic capabilities: Routlines versus entrepreneurial action. Journal of Management Studies, 49, 1395-1401.

21. Xiaohui, S., Feng, L., \& Chumnumpan, P. (2020). Platform development: Emerging insights from a nascent industry, Journal of Management, 47(8), 2037-2073. 